\title{
Complex valve reconstruction in isolated tricuspid valve endocarditis
}

Gennady V. Atroshchenko, MD, Daniel E. Munoz, MD, Jama Jahanyar, MD, PhD, Gebrine el Khoury, MD, and Laurent de Kerchove, MD, PhD, Brussels, Belgium

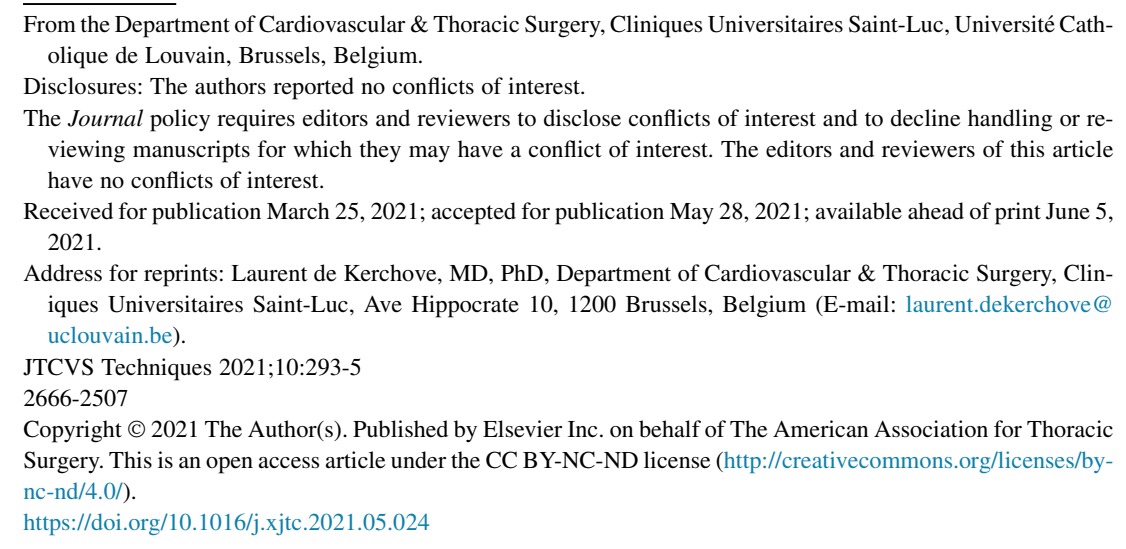

Video clip is available online.

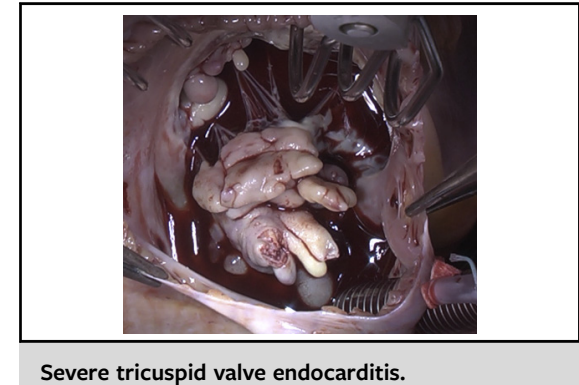

Severe tricuspid valve endocarditis.

CENTRAL MESSAGE

Complex tricuspid valve repair is feasible, even in severe endocarditis with destruction of large portions of leaflet and/or subvalvular apparatus.

See Commentaries on pages 296,298 , and 300
Right-sided infective endocarditis (IE) accounts for 5\% to $10 \%$ of all endocarditis cases. In this setting, the tricuspid valve is the most commonly affected valve, and intravenous drug abuse is the leading cause for right-sided IE. ${ }^{1}$

Whenever surgical treatment is indicated, valve repair is preferred over valve replacement. ${ }^{2}$ To reduce the risk of recurrent IE following surgical therapy, the use of foreign material during surgery should be kept to a minimum. This is particularly important in chronic intravenous drug abusers. ${ }^{1}$ A recent meta-analysis comparing valve repair with replacements demonstrated greater freedom from recurrent IE, as well as freedom from permanent pacemaker implantation in the repair group. ${ }^{3}$ Thus, valve repair is the preferred approach for patients with tricuspid valve IE.

However, in patients with extensive endocarditis, complete removal of all infected tissues can also lead to removal of large parts of native valve tissues and subvalvular apparatus. Therefore, complex reconstruction is often needed to preserve the tricuspid valve in cases of extensive valvular destruction, rendering the repair challenging. ${ }^{4}$

Herein, we present a case of severe tricuspid valve endocarditis with destruction of large parts of native tricuspid valve tissues, and our valve-sparing repair approach.

\section{CLINICAL SCENARIO}

A 34-year-old man with a past medical history significant for intravenous drug abuse was admitted to the hospital with progressive dyspnea and workup for sepsis. A computed tomography scan of the chest and abdomen identified right lower lobe subsegmental emboli in the lungs. There was also significant hepatosplenomegaly present. Blood cultures were positive for Staphylococcus aureus and Streptococcus anginosus, and on transthoracic echocardiography, a large vegetation on the septal leaflet of the tricuspid valve was identified measuring $23 \times 21 \mathrm{~mm}$ in size (Figure 1, $A$ ), with mild tricuspid regurgitation. No additional valvular lesions were identified. Biventricular function was preserved with normal pulmonary pressures. Due to persistent bacteremia and large size of the lesion, the decision for surgery was made.

\section{OPERATIVE TECHNIQUE}

On cardiopulmonary bypass, with central aortic and bicaval cannulation, the right atrium was opened, after cardioplegic arrest with normothermic blood cardioplegia was achieved. The intraoperative assessment of the valve revealed large ulcerating vegetations involving large 

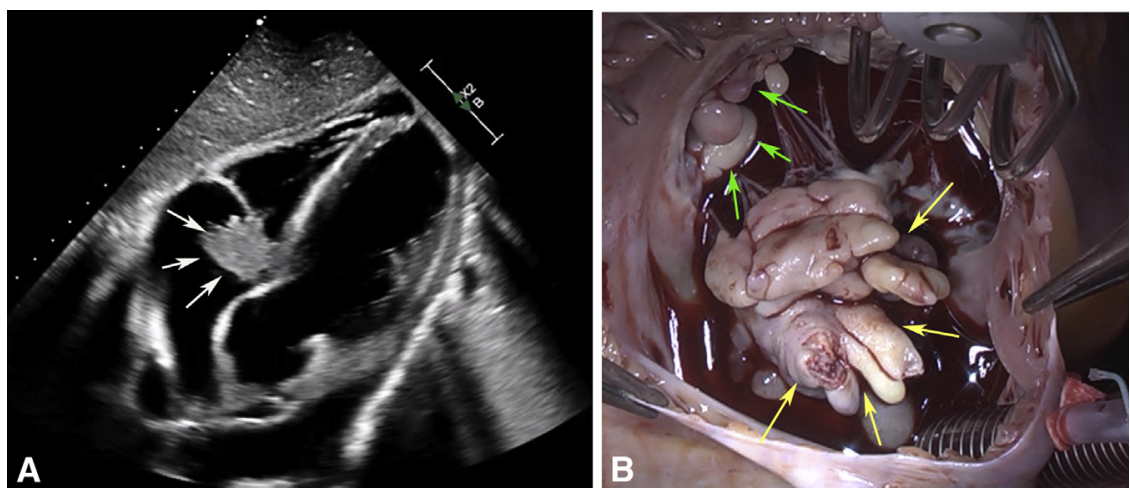

FIGURE 1. A, Preoperative echocardiograph demonstrating large vegetations on the tricuspid valve. B, Intraoperative assessment reveals large sessile vegetations on large parts of the septal leaflet (yellow arrows), and anterior tricuspid valve leaflet (green arrows).

parts of the septal and anterior tricuspid valve leaflets (Figure 1, B). Despite the extensive destruction of the valve leaflets, a valve-preserving approach was undertaken (Figure 2, A). The vegetations were resected, including large portions of the septal and anterior leaflets. This also included attached chordae tendinae on the septal leaflet.

The defects in the septal and anterior leaflets were patched (Figure 2, B), utilizing a decellularized equine pericardial patch (Matrix Patch; Autotissue, Berlin, Germany). This patch is approved in Europe for intra- and extracardiac use in children and adults. At our center, we like using this patch because it is thin and more pliable than autologous or other xenopericardial patches; thus, easier to handle, especially in tricuspid valves where the leaflets tend to be thinner. Long-term data are limited. ${ }^{5}$

In the setting of a destroyed anterior and septal leaflet, the most important leaflet to consider is the anterior leaflet. It is important to size the anterior leaflet patch in a fashion that allows for the leaflet free margin to reach the posterior/septal annulus. This is somewhat identical to the mitral valve. When the septal leaflet is patched, we try to re-create the original size as close as possible, but this is not as critical for the tricuspid valve function as is the anterior leaflet. To identify the size of the tricuspid valve defects, the leaflets should be slightly stretched to recognize the true size of the defects in the leaflets. The patch is then positioned along the rim of the leaflet defects, adding a 3- to 5-mm overlap, which adds space for a suture line.

When feasible, chordae tendinae were preserved, but in areas where the chords had to be removed, the leaflets were supported with Gore-Tex (W.L. Gore \& Associates, Newark, Del) neochords, following the basic principles also applied to the mitral valve. The neochords are adjusted to place the free margin at the level of the tricuspid valve annulus. We anchor the sutures with a figure 8 in the papillary muscle and place the 2 ends through the leaflet and lock the sutures onto themselves by going around the free margin again. With this, the free margin is fixed but can still be adjusted (during a saline test for instance) as needed before tying the sutures.

Nevertheless, a few features unique to the right ventricle and tricuspid valve are the small and not-well-defined papillary muscles. Thus, ventricular anchoring of the neochords can be trickier and challenging at times. Accordingly, in the absence of well-defined papillary muscles, we sometimes anchor the chords to the ventricle wall itself, at the level where other cords arise.
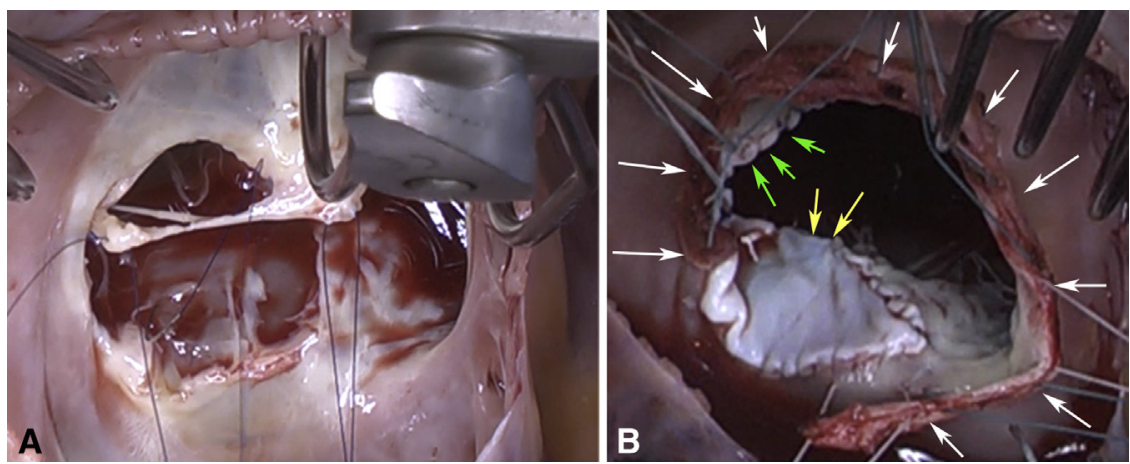

FIGURE 2. A, Intraoperative image demonstrating removal of tricuspid vegetations with concomitant removal of large parts of the anterior and septal tricuspid leaflets. B, Repair of tricuspid valve with equine pericardial patch and Gore-Tex (W.L. Gore \& Associates, Newark, Del) neochords on septal (yellow arrows) and anterior tricuspid valve leaflet (green arrows). Annuloplasty with autologous pericardial band (white arrows). 


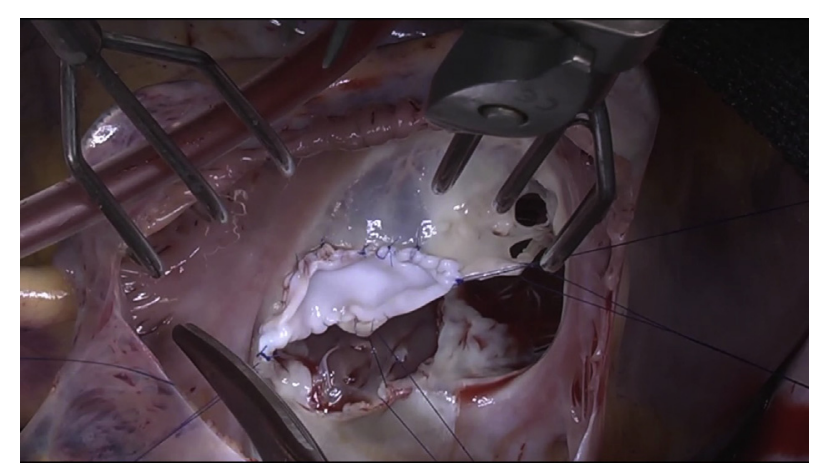

VIDEO 1. Complex valve reconstruction in an intravenous drug abuserelated severe endocarditis of the tricuspid valve. Video available at: https://www.jtcvs.org/article/S2666-2507(21)00391-6/fulltext.

To further limit the use of foreign material, annular support was provided utilizing a band of autologous pericardium in lieu of an annuloplasty ring. The pericardial strip is sized according to the length of the tricuspid annulus from the middle of the septal leaflet to the anteroseptal commissure in a counterclockwise manner. Cardiopulmonary support was then weaned, and transesophageal echocardiography showed a competent tricuspid valve, with only trivial tricuspid regurgitation (Video 1). Informed consent for publication was obtained from the patient.

\section{FOLLOW-UP}

The patient is now 6 months postoperation and undergoes follow-up at an outside institution. He continues to do well without any symptoms. He is still self-injecting intravenous drugs, but is followed by a special organization in Belgium that cares for drug addicts and provides sterile equipment and aids in the weaning of drugs. A recent transthoracic echocardiograph shows no residual tricuspid regurgitation or stenosis and no evidence for recurrent endocarditis with normal biventricular function.

\section{COMMENTS}

Tricuspid regurgitation, due to IE in active intravenous drug abusers, represents a clinical dilemma because recidivism in this population is frequent. A surgeon must decide to either replace the valve with a valve prosthesis in an active user, or attempt a valve repair and limit the use of foreign materials and the risk of prosthetic valve endocarditis in the future. The main principals of surgical management entail radical debridement of all vegetations and infected tissues, avoidance of implantation of prosthetic material, and elimination of valve regurgitation. In patients with a high risk of continued drug abuse, valve repair should be considered the first line of surgical management. ${ }^{6}$

Other alternatives in this patient population are more radical approaches such as possible complete valvulectomy. ${ }^{7}$ It is not an approach that we employ at our center due to possible sequalae such as ascites, peripheral edema, and right ventricular dysfunction that will mandate a prosthetic valve implantation, nonetheless.

A bicuspidization annuloplasty after complete resection of the posterior leaflet can also be performed, but this is not feasible for anterior or septal leaflet involvement. In that case, a downsizing of the posterior annulus must be performed with, for instance, a De Vega or Kay's annuloplasty. ${ }^{6}$

For our patient, we performed an annuloplasty with an autologous pericardial band, considering that the patient had no annular enlargement, significant tricuspid regurgitation, or pulmonary hypertension. Although a prosthetic ring would likely provide a better and more stable annuloplasty over time, we weighed the risk of recurrent endocarditis against the future need for a more stable ring annuloplasty. We decided to minimize the risk of recurrent endocarditis by simply utilizing an autologous pericardial band instead.

Medication compliance in this patient population can be unpredictable and unreliable, which may render anticoagulation therapy, for instance, a significant challenge. In this patient, we demonstrated that it is feasible to preserve the valve with good immediate and short-term results, despite the extensive destruction of valve tissues and subvalvular apparatus. Foreign material was limited to the equine pericardial patch only. Despite a protracted postoperative course, the patient was discharged in stable condition without the need for any further prosthetic valve-related pharmaceutical drug therapies.

\section{References}

1. Habib G, Lancellotti P, Antunes MJ, Bongiorni MG, Casalta JP, Del Zotti F, et al 2015 ESC guidelines for the management of infective endocarditis: the task force for the management of infective endocarditis of the European Society of Cardiology (ESC). Endorsed by: European Association for Cardio-Thoracic Surgery (EACTS), the European Association of Nuclear Medicine (EANM). Eur Heart J. 2015;36:3075-128.

2. Baddour LM, Wilson WR, Bayer AS, Fowler VG Jr, Tleyjeh IM, Rybak MJ, et al. Infective endocarditis in adults: diagnosis, antimicrobial therapy, and management of complications: a scientific statement for healthcare professionals from the American Heart Association. Circulation. 2015;132:1435-86.

3. Yanagawa B, Elbatarny M, Verma S, Hill S, Mazine A, Puskas JD, et al. Surgical management of tricuspid valve infective endocarditis: a systematic review and meta-analysis. Ann Thorac Surg. 2018;106:708-14.

4. Renzulli A, De Feo M, Carozza A, Della Corte A, Gregorio R, Ismeno G, et al Surgery for tricuspid valve endocarditis: a selective approach. Heart Vessels. 1999;14:163-9.

5. Elassal AA, Al-Radi OO, Zaher ZF, Dohain AM, Abdelmohsen GA, Mohamed RS, et al. Equine pericardium: a versatile alternative reconstructive material in congenital cardiac surgery. J Cardiothorac Surg. 2021;16:110.

6. Akinosoglou K, Apostolakis E, Koutsogiannis N, Leivaditis V, Gogos CA. Rightsided infective endocarditis: surgical management. Eur J Cardiothorac Surg. 2012;42:470-9.

7. Arbulu A, Holmes RJ, Asfaw I. Surgical treatment of intractable right-sided infective endocarditis in drug addicts: 25 years experience. J Heart Valve Dis. 1993;2 129-37. 